\title{
"
}

\section{UNA EDUCACIÓN GEOGRÁFICA PARA EL SIGLO XXI: APRENDER COMPETENCIAS PARA SER CIUDADANO EN EL MUNDO GLOBAL}

\author{
Xosé Manuel Souto González ${ }^{15}$
}

\section{RESUMEN}

La educación es una de las palabras que suelen ser invocadas por los políticos en sus programas electorales. Igualmente los medios de comunicación suelen hacerse eco de algunos informes internacionales, que además se presentan como si de una competición deportiva se tratara. Las familias suelen quejarse de la manera de educar a sus vástagos y los profesores del poco reconocimiento que tiene su labor. Y si no fuera poco, la Unión Europea nos recuerda que debemos afrontar la formación permanente, ya que es necesaria para los retos de la competitividad. La educación en los países iberoamericanos es una preocupación compartida.

Palabras clave: Didáctica de la geografía, educación social, ciudadanía.

\begin{abstract}
Education is one of the words politicians usually call in their election programmes. The Media often publish international reports about it. And they do it as if it where a sports competition. Families usually complain about how their children are educated, and teachers feel their work is not recognized. In addition, The European Union reminds us of our need of facing a continuous training as something we should do for the challenges of competitiveness. Education in Latin America countries is a concern everybody shares.
\end{abstract}

Key words: Geography Teaching, Social Education, Citizenship.

\section{INTRODUCCIÓN}

¿Qué sucede para que tantas personas e instituciones hablen de la educación y al mismo tiempo se tenga la percepción de que esta empeora? Numerosos alumnos fracasan en sus aprendizajes y un número significativo abandona sus estudios cuando desaparece la amenaza de la obligatoriedad. ¿Por qué se resisten a aprender los niños y adolescentes en una sociedad que dice llamarse del conocimiento? ¿Qué sucede para que estas personas no quieran aprovechar su derecho a una educación gratuita cuando otros niños son explotados por no poder acudir a un centro escolar? ¿Por qué no se abordan los problemas ambientales del planeta desde la lógica del conocimiento geográfico esco-

15 Profesor del Master de Formación del profesorado de Educación Secundaria de la Universidad de Valencia, del departamento de Didáctica de las Ciencias Sociales y Experimentales. 
lar? ¿Por qué no se incorporan las preocupaciones cotidianas como objeto de estudio a la geografía escolar? Estas preguntas no pueden ser abordadas desde una simple comunicación, pero sí muestra la intención de un proyecto de debate más ambicioso que estamos llevando a cabo con colegas de países iberoamericanos ${ }^{16}$.

Cabe preguntarse si la investigación realizada en los departamentos universitarios no es relevante para las necesidades que tienen los profesores de geografía y ciencias sociales en las etapas de la educación básica. Se puede plantear así una doble hipótesis: por una parte que se ha abierto una cesura entre las investigaciones universitarias en didáctica y las necesidades del profesorado en los programas de formación básica; por otra, que la difusión del conocimiento geográfico no llega a las aulas de la educación básica.

El presente artículo es una re-elaboración y actualización de la comunicación que en su día presenté al X Coloquio Internacional Geocrítica ${ }^{17}$. En dicho evento, celebrado en el año 2008, quise realizar una síntesis sobre los trabajos que se habían publicado básicamente en este portal electrónico, que constituye una espléndida referencia para cualquier investigador de las ciencias sociales. Ahora lo que pretendo es complementar dichos trabajos con los realizados por otros grupos, sobre todo en la Península Ibérica (como consecuencia de mis limitaciones intelectuales), para poder explicar la ruptura de dos tradiciones educativas, aquella que parte de los problemas prácticos del aula y otra que programa innovaciones didácticas desde las teorías bibliográficas y desde algunas investigaciones universitarias.

Aunque pueda parecer una obviedad es evidente que al profesorado de enseñanzas básicas (Educación Primaria y secundaria Obligatoria en España, desde los seis a los dieciséis años) no le parecen relevantes las investigaciones que se realizan en las universidades. Las preocupaciones del docente estriban en dos aspectos básicos: el control del gobierno del aula y la formación intelectual de los alumnos en unos contenidos que se consideran como importantes. Para poder explicar los cambios en el comportamiento del alumnado y para adoptar decisiones respecto a la selección de los contenidos de la nueva sociedad de la información no encuentran ayudas suficientes en las investigaciones realizadas. Entiendo que el gremialismo existente en las universidades, entre las Didácticas Generales y las Específicas, es un obstáculo muy importante para favorecer la necesaria relación entre ambas comunidades de investigadores y docentes.

A mi modo de ver la investigación realizada sobre la enseñanza de la geografía ha oscilado entre un estudio de los condicionantes del aprendizaje del alumnado, con sus estrategias didácticas consecuentes, y el análisis de los factores que explican la educación en un sistema institucional regulado por los poderes políticos y las leyes del mercado. A lo largo de este artículo quiero exponer algunos resultados empíricos que avalan este desarrollo, que sin embargo no son capaces de penetrar en el interior de las formas de pensar los profesores de educación básica en los momentos en que programan sus clases, las ejecutan con sus actividades o las evalúan. Por eso entendemos que la alternativa

16 Para entender mejor estas preocupaciones iberoamericanas se puede consultar en el portal Geocrítica (http:// www.ub.edu/geocrit/menu.htm) la ventana del Geoforo Iberoamericano de Educación (http://www.geoforo. $\underline{\mathrm{com} /)}$.

17 SOUTO GONZÁLEZ, X. M. Una educación geográfica para el siglo XXI: aprender competencias para ser ciudadano en el mundo global. Diez años de cambios en el Mundo, en la Geografia y en las Ciencias Sociales, 1999-2008. Actas del X Coloquio Internacional de Geocrítica, Universidad de Barcelona, 26-30 de mayo de 2008. <http://www. ub.es/geocrit/-xcol/19.htm> 
de un proyecto curricular es la que mejor aúna los esfuerzos de la investigación con la innovación didáctica. Una alternativa que pensamos desarrollar en el punto final de este artículo.

Lo primero que nos podemos preguntar, como en su día lo hizo el profesor Horacio Capel, es para qué se estudia geografía en el sistema escolar y cuál es su utilidad en una formación básica ciudadana. Según su opinión la geografía debía "seguir estudiándose por tres tipos de razones: 1) porque ya existe y es una ciencia con una larga y rica tradición intelectual; 2) porque es una disciplina con un gran valor educativo y formativo; y 3) porque hay problemas que la geografía, es decir los profesionales formados en el campo de la geografía, puede ayudar a estudiar y resolver" ${ }^{\prime 1}$. Son afirmaciones de deseamos cuestionar a lo largo de esta comunicación.

Algunas de sus argumentaciones, como la del coste de esta materia frente a la disgregación de las ciencias sociales, nos hace reflexionar sobre las decisiones administrativas para organizar el curriculum, que tiene una raíz claramente histórica y está relacionada con las metas que establecen los grupos sociales en el poder ${ }^{19}$. Igual sucede con lo que se considera "nociones valiosas" en el campo de la educación de un país y en la "toma de conciencia de los problemas del mundo contemporáneo". En todas estas situaciones incide la concepción que del mundo tienen los docentes y, sobre todo, quienes los forman en las universidades y quienes planifican los contenidos educativos.

En efecto, la pregunta sobre el sentido educativo de la geografía tiene un contenido claramente histórico. La educación se inserta en el marco de la institucionalización del sistema escolar. Así podemos diferenciar sus orígenes en el siglo XIX, con la organización de los estados liberales y su adecuación al sistema de grados (Primaria, Secundaria, Universidad) a lo largo de los siglos XIX y XX. La ya tópica diferenciación entre la educación de elites y de masas se sigue refiriendo a los sistemas escolares de niños y adolescentes, para dar cuenta de la prolongación de la enseñanza obligatoria hasta los 14 y 16 años de edad; ya se han puesto en marcha las políticas de formación continua y para el empleo (a lo largo de la vida) después de la publicación de las oportunas normas legislativas en 2007 y 2008 en España. En el contexto de la crisis económica en España los cursos de formación para personas desempleadas son muy numerosos y la formación para alcanzar niveles mínimos de escolaridad ya son una realidad. En España la ley de formación para el empleo indica que todos los trabajadores deben alcanzar como mínimo el Graduado en Educación Secundaria Obligatoria. Por tanto se han dado los cambios, se mantienen y amplían las expectativas.

Por eso qué geografía enseñar es una cuestión que afecta a los individuos y suele ser decidida en las estructuras del poder administrativo ${ }^{20}$ en las cuales existen diferentes y eficaces cauces para que las ideas emanadas en los grupos hegemónicos puedan llegar a las aulas de personas menos poderosas. Sin embargo, la mayoría del profesorado de la enseñanza básica tiene poco tiempo para debatir y pensar cómo llegan hasta las puertas de sus aulas los contenidos educativos. No suelen preguntarse sobre el origen de la organización curricular; simplemente se trata de desarrollarla en el aula. En este sentido, la investigación educativa tiene un reto evidente: mostrar cómo las decisiones de qué enseñar y para qué educar tienen unas implicaciones políticas y éticas, que implican a la

18 Nos referimos al trabajo del profesor Horacio Capel (1998; pp. 1-2)

19 Precisamente el Geoforo recoge en su Foro 7, un amplio debate sobre la organización escolar entre ciencias sociales y geografía. Ver nota 1 para referencia del Geoforo.

20 Ver el trabajo de A. Luis, y J Romero (2007) y su reseña de este libro en: Souto González, X.M. (2007) en la revista Biblio $3 W[$ http://www.ub.es/geocrit/b3w-764.htm]. 
labor del profesorado. Sin embargo, al menos desde España, podemos constatar que dicha difusión de este conocimiento político no ha llegado hasta los docentes de la enseñanza básica. Es un asunto que no suele formar parte de los programas de formación docente y que tampoco forma parte de los debates del profesorado.

Y los responsables de organizar los sistemas escolares plantean esta pregunta en el conjunto de normas morales y de funcionamiento que regulan las relaciones de enseñanza y aprendizaje en el seno de una sociedad. Pero no todo está controlado por las decisiones emanadas desde el poder. También existen resquicios para la "emancipación" y creatividad. A este respecto lo que piensan los gremios de educadores y científicos puede ser de utilidad para organizar programas didácticos y materiales educativos. Por eso la educación es una actividad que libera temores y castiga actitudes de rebeldía. La autonomía del profesor en el aula para adoptar decisiones en relación a la metodología y a la selección de contenidos es un asunto relevante que todavía no ha sido bien desarrollado por la comunidad de investigadores de las ciencias sociales. No es suficiente con un estudio genérico, es necesario (tal como en las superposiciones cartográficas) descender a análisis de casos individuales.

En este artículo vamos a intentar ofrecer un panorama de los retos educativos que tiene la educación geográfica al final de este primer decenio del tercer milenio. La posibilidad de conocer qué se hace en diferentes lugares, las innovaciones tecnológicas en la difusión y gestión de la información, los propios avances en el conocimiento científico y la valoración de las experiencias realizadas en la didáctica del aula nos permitirán avanzar en la definición de líneas alternativas para que la tarea educativa geográfica sea posible en el siglo XXI. Pero también habrá que conocer las percepciones y expectativas de niños, adolescentes, jóvenes y adultos. Ellos también tienen su concepción acerca del valor educativo de la geografía para su formación, sea ésta un medio para obtener una acreditación profesional, una satisfacción a su curiosidad, o una manera de relacionarse con otras personas.

Hay dos campos de desarrollo curricular donde el conocimiento geográfico tiene un valor incuestionable. En primer lugar en la definición del marco geográfico y el contexto social donde se desarrolla la acción educativa; o sea, el proyecto educativo del centro. La geografía puede colaborar activamente en el análisis de las características y factores que condicionan el aprendizaje del alumnado: sus deseos, expectativas sociales, valores culturales dominantes, relaciones de sus viviendas con el sistema urbano dominante o las relaciones sociales y familiares que determinan las emociones, afectos y razonamientos del alumnado. Una tarea poco desarrollada por lo general, pese a que se reivindica la autonomía de los centros escolares.

En segundo lugar, la instrucción o la formación en los contenidos programados dentro de la materia o en el conjunto de las ciencias sociales y naturales. La enseñanza de conceptos, habilidades y actitudes en la educación geográfica ha ido cambiando a lo largo de los últimos diez años. Los diversos artículos que se han publicado en las páginas de las revistas electrónicas de la web de Geocrítica así lo acreditan. Nuestro deseo es organizar esta información y plantear algunas hipótesis que permitan indagar sobre las posibilidades de una educación geográfica que facilite la comprensión y explicación de los problemas ambientales y sociales en el mundo presente.

Además de las secciones de Biblio3W, Scripta Nova, Aracne y Geoforo de Educación, del portal Geocrítica, hemos consultado la página web de la asociación de geógrafos españoles (AGE, Grupo de 
Didáctica de la $A G E$ ) para conocer los contenidos de los diferentes congresos celebrados ${ }^{21}$, así como las revistas específicas de didáctica de las ciencias sociales (Iber, Didáctica Geográfica) y la producción de grupos como Asklepios, Cronos, Garbi, Gea-Clio, IRES o Insula Barataria, que han tenido un eco importante en la renovación de la didáctica de las ciencias sociales en España.

\section{LOS OBJETIVOS DE LA EDUCACIÓN GEOGRÁFICA DESDE LA INVESTIGACIÓN UNIVERSITARIA}

Como hemos indicado, las finalidades de la educación geográfica no vienen determinadas por las necesidades sentidas por la población escolar, sean profesores o alumnos, sino básicamente por la interpretación que de ellas hacen las autoridades legislativas y ejecutivas. En este sentido, como han mostrado los trabajos de Sergio Claudino ${ }^{22}$ y Julia Melcón ${ }^{23}$, la implantación de la geografía en el sistema escolar fue tardía en el caso de la Educación Primaria, pues su función era la de suministrar conocimientos culturales, enciclopédicos, a las elites gobernantes y a sus descendientes. En el caso de España correspondía con la Educación Primaria Superior, un nivel educativo con unos contenidos que a juicio de los legisladores del Estado liberal decimonónico "no eran indispensables para las clases pobres".

Además queremos subrayar que estas aportaciones desde el análisis de los contenidos, que se han desarrollado en los programas y manuales escolares de la geografía, han incidido en la identidad territorial. Bien sea desde una óptica conservadora o desde planteamientos más innovadores, ligados, en el caso de España, a la Institución Libre de Enseñanza. La ILE y la Segunda República se relacionaron con la Escuela Nueva y hacen propuestas muy relevantes desde centros de Educación Primaria de diferentes provincias ${ }^{24}$. La influencia de los contextos administrativos es muy importante para explicar las transformaciones que se pueden producir en el seno del sistema escolar, como fue en su momento la oportunidad aprovechada por Torres Campos en la formación de las maestras, cuando las mujeres docentes no tenían la misma consideración que el varón. O bien la posibilidad de organizar nuevos programas de enseñanza de la geografía, como sucedió en el franquismo con los Institutos Laborales ${ }^{25}$.

Es decir, a lo largo de los siglos XIX y XX se va constituyendo una "manera natural" de entender el conocimiento geográfico escolar, que es muy semejante para los dos Estados liberales de la península ibérica, como mostramos en su día siguiendo los estudios de Claudino y Melcón. La relativa

21 Quiero hacer explícita la ayuda recibida de María Jesús Marrón Gaite, presidenta durante muchos años del Grupo de Didáctica de la AGE, en el envío de los materiales editados por la Asociación.

22 Sobre la Tesis doctoral de Sérgio Claudino (2001) puede encontrarse un resumen y comentario en: X. M. Souto González en Biblio 3W, [ http://www.ub.es/geocrit/b3w-379.htm]

23 Julia Melcón Beltrán (1989) estudió las relaciones entre la geografía y la formación de maestros, así como los fundamentos de la enseñanza de la geografía en España (Melcón,1995, páginas 147 a 200).

24 Una referencia a este modelo de escuela, que se entendía como moderna, alegre y activa la hemos observado en la figura de Luis Barreiro (1936). Sus propuestas didácticas están en consonancia con las que se han realizado en los años ochenta del siglo XX y a su vez se relacionaban con las realizadas en algunas partes de España y Francia por las mismas fechas de los años treinta del siglo pasado.

25 Sobre este asunto concreto podemos consultar la Tesis doctoral de José Villanueva, posteriormente publicada (Villanueva, 2003). Una reseña crítica la podemos encontrar en la revista electrónica Biblio3W n ${ }^{\circ} 320,28$ de octubre de 2001, de autoría del profesor Horacio Capel [http://www.ub.es/geocrit/b3w-320.htm] 
autonomía de los autores de los libros de texto, estudiada en el caso lusitano por el citado profesor Claudino, da paso a una etapa en la cual existe un mayor control desde las instancias administrativas. Las vinculaciones entre los poderes eclesiásticos, las sociedades geográficas, la expansión comercial y la educación ha sido mostrada en diferentes trabajos de la historia de la geografía, que no es posible referir aquí por falta de espacio.

Mi experiencia en formación inicial del profesorado, de Ciencias Sociales en Secundaria, me ha hecho consciente de esta deficiencia. Los licenciados, o graduados en Historia, Geografía e Historia del $A_{r t e}{ }^{26}$ tienen una carencia enorme de conocimientos sobre la institucionalización de estas materias en el sistema político de los Estados constitucionales o liberales. Y aquí aparece otro reto en la organización de los planes de estudio de los Grados de Geografía. Una situación que se repite en otros países iberoamericanos, como sabemos por las comunicaciones mantenidas en el Geoforo.

Por esta misma razón interesa dar cuenta de la recepción de este tipo de conocimiento en las tierras iberoamericanas, en los momentos de independencia de las metrópolis ibéricas. Así en el caso de la educación escolar venezolana, José A. Santiago nos subraya la incidencia de este tipo de geografía, que sin duda entraría de la mano de los grupos criollos dominantes. Así nos hace ver la importancia que ha tenido en el desarrollo histórico de la geografía escolar venezolana la enseñanza a través de cuestionarios, como fue el Catecismo de la Geografia de Venezuela para el uso de las escuelas primarias (1841), redactado por Agustín Codazzi, (que) representó una evidencia concreta de la necesidad existente (...) de proponer una enseñanza de la geografía nacional, para contrarrestar los compendios enviados desde Europa"27.

En las postrimerías del siglo XIX e inicios del XX se buscaron algunos modelos alternativos a esta manera de entender la enseñanza, que básicamente provenían de posiciones burguesas ilustradas que buscaban salir del control académico y moral de los poderes conservadores. Además la geografía se incorporaba a la Educación Primaria, que se establecía en el conjunto del sistema escolar, que adoptaba un sistema de grados. Las relaciones entre una orilla y otra del Atlántico serán reforzadas en el siglo XX a través del constante trabajo de personas como Pau Vila, que en su exilio posterior a la guerra civil desarrolló sus ideas en Colombia y Venezuela.

Como podemos apreciar, el conocimiento que empezamos a disponer de la evolución histórica de la enseñanza de la geografía en los distintos países iberoamericanos nos pone de relieve algunas semejanzas, que no son sólo respecto a la metodología didáctica en abstracto, sino que tienen profundas implicaciones en la difusión de una cultura geográfica, lo que determina la comprensión del mundo presente: sus conflictos o el orden "natural". Si un profesor de la enseñanza básica no tiene oportunidad de cuestionarse la difusión de este conocimiento en su formación inicial, es posible que a lo largo del ejercicio de su docencia no vuelva a disponer de un momento para replantear el origen de los contenidos que imparte en el aula. Resulta difícil, por no decir imposible, que un profesorado que no tiene la capacidad crítica para analizar la autonomía de sus decisiones pueda enseñar a un pupilo a desarrollar su capacidad de autonomía intelectual.

26 En España desde los años ochenta del siglo XX las carreras de Historia, Historia del Arte y Geografía son independientes. De tal manera que pueden licenciarse o graduarse en Historia sin haber cursado casi ningún contenido específico de Geografía, y viceversa. Una situación que no se puede transformar en un curso monográfico del Master en Secundaria.

27 La evolución histórica de la enseñanza de la geografía en Venezuela la hemos consultado en José A. Santiago (consulta on-line: http://hum.unne.edu.ar/revistas/geoweb/indnurev.htm) 
En este sentido, nos parece que una buena estrategia de formación inicial del profesorado estriba en el estudio de la institucionalización de la disciplina como materia escolar. Pero para ello es preciso relacionar el presente con el pasado. Es decir, cuestionarse la heterogeneidad, conflictos y cultura escolar que predomina en el momento presente y buscar los argumentos explicativos en el pasado. Un profesor que identifica los problemas presentes y posee recursos explicativos para entenderlos en un momento histórico es un docente con más seguridad en sus actuaciones. Y sin dicha estabilidad emocional es muy difícil ejercer la tarea educativa.

En consecuencia, las distintas concepciones del profesorado sobre la evolución histórica de la materia adquieren el sentido más profundo en la comunicación que se establece con el alumnado. Entonces es cuando las finalidades y criterios de evaluación se transforman en actividades didácticas; pues bien, para poder organizar éstas de una manera coherente es necesario conocer con rigor las estrategias docentes y las metodologías de aprendizaje. En caso contrario es muy probable que dominen no sólo las rutinas, sino también una concepción autoritaria del saber.

\section{LA BÚSQUEDA DE ALTERNATIVAS DIDÁCTICAS DESDE LA ENSEÑANZA BÁSICA}

Las aportaciones de la pedagogía (Diseño curricular, transversalidad, competencias) y de la psicología (Constructivismo, aprendizaje significativo) han hecho pensar a muchas personas, entre las que me encuentro, que era posible organizar una alternativa a la educación geográfica desde su didáctica. Entendíamos que la mayor parte del tiempo transcurre en las aulas y que, por eso mismo, era imprescindible un análisis de la comunicación interpersonal en la construcción social del conocimiento. La comunicación educativa supone un análisis de las relaciones de poder en un marco institucional (la institución escolar) y considerar cuáles son las expectativas y valores que le conceden las personas (profesores y alumnos) al saber.

Mantenemos que, desde enfoques analíticos de la realidad del aula, es posible plantear la hipótesis de organizar la didáctica con un modelo contra hegemónico. Eso es lo que a nuestro juicio manifestó el grupo IRES, que buscó la manera de configurar un proyecto alternativo a través de la consideración de la labor investigadora del maestro en el aula ${ }^{28}$. Se buscaba que el núcleo desde el cual cambiar la enseñanza no dependiera de elementos extraños a las relaciones de la comunidad escolar, sino que desde las investigaciones escolares, con ayudas externas, fuera posible abordar los problemas que preocupaban a profesores y alumnos. Por eso le han concedido una gran importancia a la definición del conocimiento escolar, que se sitúa en la encrucijada entre el académico racional y el espontáneo y vulgar.

Como se pudo apreciar en un reciente Congreso sobre Redes de Innovación en España existen grupos que relacionan sus propuestas de mejora en el aula con el análisis de la escuela como institución, frente a otros que sólo se dedican a abordar problemas concretos ${ }^{29}$. Pero en todos los casos

28 Un amplio resumen del proyecto IRES se puede consultar en F.F. García y R. Porlán en la revista electrónica Biblio 3, n²05. (http://www.ub.es/geocrit/b3w-205.htm) de 16 de febrero de 2000

29 Nos referimos al Congreso de Redes de Innovación Entretod@s celebrado en Madrid los días 1, 2 y 3 de febrero de 2008, al cual fueron invitados todas redes o grupos de innovación, desde Movimientos de Renovación Pedagógica a otros colectivos con finalidades más específicas. Posteriormente se celebró un segundo Congreso los días 11, 12 y 13 de diciembre de 2009 en la ciudad de Cuenca. Un resumen de lo tratado en dichos Congresos se puede consultar en el Portal Innova (http://www.portalinnova.org/). 
subyace el interés de ofrecer soluciones a los problemas que surgen en el aula, que no son solo de conocimiento, sino también de comportamiento y disciplina. En este mismo sentido debo hacer referencia a la evolución del proyecto Gea-Clío, pues surge de los mismos intereses aquí indicados ${ }^{30}$. Por el contrario lo que queremos dejar constancia es que en el mismo ámbito de la didáctica de la geografía otras personas han realizado esfuerzos semejantes.

Así tenemos que destacar el trabajo que desde les Illes Balears ha realizado con su tesis doctoral A. Ballester, para quien la situación actual de la didáctica de la geografía en las aulas está basada mayoritariamente en la repetición de hechos y conceptos. Por eso como alternativa ha planteado la necesidad de encontrar nuevas maneras de trabajar teniendo en cuenta las características del alumnado que al tiempo permitan un aprendizaje más coherente de esta disciplina. Una forma de entender la didáctica que ha traspasado las fronteras disciplinares, como se puede observar en la página web del aprendizaje significativo que ha sido adaptado por la Consejería de Canarias ${ }^{31}$.

Igualmente destacamos la aportación de la profesora colombiana Elsa A. Rodríguez Moreno, que relaciona el aprendizaje de los alumnos con la formación del profesorado ${ }^{32}$. Entiende que dadas las carencias de la formación del profesorado, las rutinas en la aplicación del curriculum y las deficiencias en el aprendizaje del alumnado es preferible iniciar por este camino la propuesta de innovación. Así surge su propuesta para desarrollar "una pedagogía conceptual de la geografía", que supone la gradación de ejercicios de diferente complejidad en relación directa con las edades del alumnado.

También en esta misma línea de investigación queremos destacar el trabajo de Francisco García, pues en su Tesis doctoral muestra la gradación existente en las ideas del alumnado desde una visión simple y armónica del medio urbano hasta otra más compleja, analítica y donde es posible percibir ideas que surgen como factores explicativos. En su estudio queda clara la importancia de los conceptos aportados por la enseñanza escolar, pero también confirma la gran riqueza y variedad de concepciones de los alumnos sobre el medio urbano, en las que están presentes "componentes del conocimiento cotidiano y de conocimiento escolar que configuran sistemas de ideas que, en todo caso, presentan una lógica peculiar" ${ }^{33}$.

30 Una valoración y resumen de la historia del proyecto Gea-Clío se puede ver en: LLÁCER PÉREZ, Vicent. Innovación didáctica y cambios educativos en España. El Proyecto GEA-CLÍO. Diez años de cambios en el Mundo, en la Geografia y en las Ciencias Sociales, 1999-2008. Actas del X Coloquio Internacional de Geocrítica, Universidad de Barcelona, 26-30 de mayo de 2008. <http://www.ub.es/geocrit/-xcol/20.htm>

31 Se puede consultar su tesis doctoral en la publicación posterior (A. Ballester, 1999). Una reseña de dicho libro se encuentra en Biblio 3W. (www.ub.es/geocrit/b3w-237.htm), No 237, 6 de junio de 2000. En la página web: www. aprendizajesignificativo.com se hace referencia al seminario organizado en la isla de Tenerife, con el apoyo del gobierno canario: "En la búsqueda de dar respuesta a esta preocupación, nos encontramos con un libro digital titulado "Aprendizaje Significativo en la Práctica" de Antoni Ballester en el que se relataba el proceso seguido en seminarios de formación acerca del aprendizaje significativo. Este libro fue el eje del proceso de las reuniones de coordinación formación mensual de los/as coordinadores/as, desarrollando el libro, respetando la dinámica planteada en el mismo".

32 E.A. Rodríguez de Moreno (2000). Una reseña de este trabajo la he realizado en el número 292 de la revista electrónica Biblio3W (http://www.ub.es/geocrit/b3w-292.htm) del 12 de mayo de 2001.

33 El estudio teórico y empírico de F. García concluyó en su Tesis Doctoral, presentada en la Universidad de Sevilla en 1999. Una reseña de la misma la ha realizado el profesor Horacio CAPEL, en la revista Biblio 3W. Revista Bibliográfica de Geografia y Ciencias Sociales de la Universidad de Barcelona (http://www.ub.es/geocrit/b3w-194. $\underline{\mathrm{htm}} \mathrm{n}^{\mathrm{o}}$ 194, 8 de enero del 2000.Igualmente resulta de interés consultar la publicación resumida de este trabajo 
Otra manera de pensar la enseñanza de la geografía, inserta en el sistema escolar dentro de las áreas curriculares, es la que manifiesta Alfonso Guijarro ${ }^{34}$, que entiende que las reformas del curriculum son muchas veces superficiales, pues los procesos de reforma del sistema educativo han cambiado poco la situación, quizá porque seguían el modelo del conde de Lampedusa, o sea cambiar todo para que la situación siga inmutable.

No obstante, como señalamos al socaire de esta tesis doctoral, también se abrieron oportunidades al debate público sobre el papel de las disciplinas en la educación obligatoria, y más en concreto en esta materia. Como en su día manifesté era, y es, preciso investigar desde el aula los problemas concretos que se pueden analizar teóricamente, pero para ello es preciso disponer de buenos proyectos curriculares que aúnen teoría y práctica. Pues en caso contrario se corre el riesgo de acercarse a "la situación escolar desde una perspectiva de "persona ilustrada", con objetivos educativos que se perciben desde la óptica de una racionalidad "para el pueblo, pero sin el pueblo". La ausencia absoluta a los esquemas de conocimiento del alumnado creo que hacen de su debate un juego dialéctico lúcido, pero poco útil para la mejora de la enseñanza. Y tampoco existe un análisis de las decisiones que han ido tomando los profesores en la interpretación de las diversas propuestas de temarios y materiales curriculares. Mantengo con rotundidad que un estudio riguroso de los planteamientos didácticos debe llevar aparejado un examen de las expectativas y decisiones que en diversos momentos han condicionado el trabajo de los profesores en el aula" ${ }^{\prime 3}$.

Este principio educativo se ha visto reforzado con la Tesis doctoral de Enric Ramiro, que ha puesto de manifiesto la fuerte cesura que existe entre las investigaciones universitarias y su difusión entre el profesorado ${ }^{36}$. No sólo en el desconocimiento de las revistas propias, sino también en el uso de las técnicas de trabajo y metodologías de una cultura académica que por veces se aísla del entorno social que dice estudiar. Y esta ruptura del diálogo entre los niveles universitarios y básicos se observa en el uso que se hace en secundaria de las tecnologías didácticas o de materiales que no sean el libro de texto. Los datos ofrecidos en su Tesis doctoral, producto del análisis de las encuestas realizadas, así como de las lecturas de trabajos de innovación, confirmarian la hipótesis que estamos manteniendo. Las investigaciones universitarias no llegan a la opinión pública ni a las aulas de edu-

(F. García, 2003), así como el comentario que ha hecho la profesora María BLAY RUBIO sobre dicho libro en la Revista Biblio 3W n ${ }^{\circ}$ 495, 29 de febrero de 2004. (http://www.ub.es/geocrit/b3w-495.htm). En ambos casos nos encontramos con una teoría bien formulada, y construida a modo de síntesis del conocimiento del medio urbano en las aulas, y una aportación empírica, con los resultados de las encuestas del alumnado, que nos permiten indicar que estamos ante dos obras de referencia para conocer el alcance y limitaciones de este tipo de investigaciones educativas.

34 La Tesis doctoral de Alfonso Guijarro (1997), bajo la dirección del profesor Alberto Luis, inició una serie de estudios académicos que provocaron un importante debate sobre la enseñanza de la geografía en España. Así se puede entrever ya en la reseña del profesor Horacio Capel sobre esta misma obra, que se puede consultar en Biblio 3W. Revista Bibliográfica de Geografia y Ciencias Sociales, Universidad de Barcelona, no 49, 30 de septiembre de 1997 (http://www.ub.es/geocrit/b3w-49.htm), que genera la respuesta que aparece en la nota siguiente.

35 La cita procede del artículo titulado ¿Qué sentido tienen la educación geográfica e histórica en el umbral del siglo XXI?, publicado en Biblio 3W. Revista Bibliográfica de Geografia y Ciencias Sociales Universidad de Barcelona, no 63, 9 de febrero de 1998 (http://www.ub.es/geocrit/b3w-63.htm).

36 La Tesis doctoral del profesor Enric Ramiro documenta con precisión la disociación entre el conocimiento académico universitario y las necesidades percibidas por el profesorado de la enseñanza básica. Una recensión de dicho trabajo lo ha realizado el prof. Horacio CAPEL en Biblio 3W, n 107, 23 julio 1998 (http://www.ub.es/ geocrit/b3w-107.htm). Posteriormente el mismo Enric Ramiro i Roca ha profundizado sobre los aspectos didácticos de la enseñanza de la geografía (1998). 
cación básica, como también confirman los trabajos realizados en Argentina sobre la opinión vulgar que tienen los profesores y familias acerca de la geografía ${ }^{37}$.

Con todo, entendimos que era preciso delimitar el papel educativo que juegan las Tecnologías de la Información y el Conocimiento (TIC) en las estrategias didácticas del docente y en su influencia sobre una cultura cotidiana por parte del alumnado. A veces llegan a las aulas disfrazadas de recursos entretenidos y no se utilizan con su valor educativo. En este sentido queremos destacar las aportaciones de Jesús Romero ${ }^{38}$, que ha precisado el uso instrumental que predomina en las aulas de Secundaria, así como las posibilidades para innovar en la enseñanza de la historia. Esta definición teórica nos ha sido útil para constatar que las influencias de las TICs en los comportamientos de los adolescentes escolares está determinada desde las pautas de cliente mercantil y no tanto de usuario crítico, o sea de un ciudadano reflexivo ${ }^{39}$. Desde las posibilidades docentes las posibilidades que se abren para definir nuevas estrategias didácticas son importantes, como se ha hecho ver en las investigaciones particulares ${ }^{40}$

También en el caso de la geografía portuguesa he tenido la fortuna de colaborar con la profesora Alzira Santos, que en su Memoria de Licenciatura (Tese de Mestrado) nos ha ofrecido una información rigurosa de las dificultades que existen para innovar desde la práctica del aula. Por eso, el conocimiento de la realidad ambiental suele ser superficial, pues adolece del tiempo y la formación precisa para abordar una investigación que convierta la necesidad de innovar en una práctica investigadora ${ }^{41}$. En todos estos casos lo que presenciamos es que la investigación educativa necesita disponer de datos empíricos sobre la realidad del aula, donde conviven alumnos y profesores sujetos a unas normativas sociales (horarios, disposición de aulas y recursos, programación de actividades, normas de comportamiento...) que influyen decisivamente en las tareas didácticas. Olvidar este hecho, o minusvalorarlo, es cometer un inmenso error en el diagnóstico de la situación educativa de un centro escolar o de un país. No se trata de reivindicar el nivel micro de la educación, sino de hacer evidente que sin las consideraciones que hemos realizado sobre alumnos y profesores es imposible analizar educativamente las transformaciones y las rutinas.

En este ámbito de dificultades adquiere más valor el análisis que se puede hacer de las técnicas de trabajo, como las cartográficas, que han sido objeto de estudio por parte de la profesora $\mathrm{M}^{\mathrm{a}}$

37 FERNÁNDEZ CASO, María Victoria; GUREVICH, Raquel; SOUTO, Patricia; BACHMANN, Lía; AJÓN, Andrea; QUINTERO, Silvina. La imagen pública de la geografía. Una indagación desde las visiones de profesores y padres de alumnos secundarios. Biblio 3W. Revista Bibliográfica de Geografia y Ciencias Sociales, Universidad de Barcelona, Vol. XV, no 859, 15 de febrero de 2010. <http://www.ub.es/geocrit/b3w-859.htm>. [ISSN 11389796].

38 La Tesis doctoral de Jesús Romero (1998) y la reseña del profesor Horacio Capel en Biblio $3 W$, no 101 , 9 de julio 1998 (http://www.ub.es/geocrit/b3w-101.htm) inciden en el debate anunciado en notas precedentes (ver notas 17 y 18$)$.

39 Un estudio empírico sobre las percepciones de los adolescentes respecto al uso social y educativo de las TIC lo hemos realizado en el trabajo interdisciplinar de J. Sobes, X.M. Souto y N. Traver (2004)

40 Ver el estudio realizado por J. Miquel y E. Alegre (2004)

41 Véase al respecto el documentado trabajo de Alzira Santos Silva (2000), así como la reseña que yo mismo he realizado en Biblio 3W. Revista Bibliográfica de Geografia y Ciencias Sociales, Universidad de Barcelona (http://www. ub.es/geocrit/b3w-243.htm). 
Luisa D'Angelo en estudios comparativos entre Argentina y España ${ }^{42}$. Sobre todo por un motivo escolar importante, como es que los mapas sintetizan una información simbólica que no suele ser desentrañada por los alumnos, así como por la dificultad que éstos tienen para expresar sus ideas cartográficamente. En este mismo campo de estudio destacamos las aportaciones que proceden de Italia (Calandra, 2008) y que manifiestan la necesidad de disponer de un conjunto de conocimientos técnicos sobre las habilidades cartográficas, pues sin ellas es imposible desarrollar la competencia lingüística, pese a las declaraciones de buenas intenciones.

Los argumentos anteriores quieren mostrar la necesidad de potenciar la investigación didáctica desde los problemas del aula, para lo cual son necesarios proyectos curriculares, donde los fines sean correspondidos con secuencias de actividades para llevar al aula. Y además, una innovación de grupos y personas que estén relacionados con la formación del profesorado, tan necesaria y alejada de la realidad en los países iberoamericanos. En efecto, como señalan S. Claudino y A. Oliveira la formación inicial es muy desigual, pero con una característica común: su insuficiencia para hacer frente a los retos de la ciudadanía global ${ }^{43}$, donde la construcción del saber es más compleja. Un problema que todavía es más grave en el caso de España, donde los planes de formación del profesorado de Secundaria permanecen obsoletos desde los años setenta del siglo XX.

\section{LOS DESEOS DE UNA EDUCACIÓN CIUDADANA CRÍTICA}

En los últimos años, en compañía de algunos colegas de la comunidad iberoamericana, hemos querido incidir en la aportación que tiene la enseñanza de la geografía para la formación ciudadana. Al mismo tiempo no queremos rehuir del debate sobre la enseñanza de las competencias, aunque manifestemos un cierto recelo ante el uso tecnocrático de estos conceptos. Tal como en su día fue el constructivismo y los procedimientos ${ }^{44}$, este nuevo concepto puede permitir abrir un debate educativo, si sabemos huir de polémicas bizantinas sobre la terminología y nos centramos en las aportaciones que pueden hacer a la construcción del conocimiento didáctico.

Para ello es preciso cuestionar la cultura que se enseña en la escuela, como hace algunos años manifesté desde esta misma plataforma. Es necesario desenmascarar las propuestas reaccionarias y potenciar las innovadoras. Y para este menester es preciso cuestionar el papel de las familias en los centros escolares, las relaciones sociales que se establecen en su seno o los criterios de definición de la denominada calidad escolar ${ }^{45}$. Con estos mimbres hemos querido construir un proyecto curricular desde 1989 hasta este año 2010; son más de veinte años de proyecto Gea-Clío. Su trayectoria nos permite extraer algunas conclusiones que creemos que son relevantes para el futuro de la investigación e innovación en la educación geográfica.

42 Una aproximación a las ideas mantenidas por esta profesora lo he realizado en la reseña (Souto, 2006) de la revista Biblio $3 W$ Revista Bibliográfica de Geografia y Ciencias Sociales,

43 Ver Claudino y Rodrigo, 2005 en Biblio 3W, Revista Bibliográfica de Geografia y Ciencias Sociales, Universidad de Barcelona, Vol. X, no 588, 10 de junio de 2005. [http://www.ub.es/geocrit/b3w-588.htm]

44 Así lo manifestamos en nuestra propuesta inicial sobre la enseñanza de la geografía en Secundaria (Souto, 1988; 5). Frente a un marco curricular tecnocrático subrayamos la posibilidad de trabajar desde la metodología constructivista con unos objetivos sociales, como lo seguimos realizando desde entonces.

45 Una interpretación de lo que decimos la he abordado en Cultura académica, fracaso escolar y reforma de las humanidades en Scripta Nova. [http://www.ub.es/geocrit/sn-96.htm] (Souto, 2001) 
En primer lugar, es necesario indicar la probabilidad de que en este camino de la innovación hayamos sucumbido en las ingenuidades propias del currículo ideal o soñado, pero también es cierto que las ventajas de un proyecto curricular que trabajaba con las ideas de los alumnos, y con sus textos, reacciones y afectos, nos ha permitido contrastar sus resultados con el currículo retenido, no sólo a corto plazo, sino en un tiempo más largo. Así en el estudio de los recuerdos del alumnado sobre los conocimientos escolares hemos podido comprobar que no sólo se guardan en la memoria conceptos e interpretaciones de la materia, sino también de actitudes y valores transmitidos por el profesorado en su quehacer diario ${ }^{46}$.

Teniendo en consideración esta praxis de la educación geográfica encontramos en la vía del estudio de los problemas sociales una oportunidad para renovar la enseñanza y la cultura escolar desde el conocimiento geográfico. Es lo que hemos intentado desde el proyecto Gea-Clío y que parcialmente hemos expuesto en otros Congresos de Geocrítica, donde hemos tenido la oportunidad de mostrar la interpretación didáctica que nosotros hacíamos de algunos problemas ${ }^{47}$.

En esta búsqueda de alternativas a la educación geográfica el proyecto Gea-Clío entendió que era necesario relacionar las actividades didácticas con los problemas sociales y ambientales percibidos por la ciudadanía. No obstante, existía una dificultad metodológica para tratar de una manera adecuada dichos problemas en el aula. Es decir, era precisa una investigación en el campo de la didáctica. A este menester dedicamos un gran esfuerzo colectivo, con grupos de profesores y alumnos, analizando las relaciones entre técnicas de trabajo, estrategias de enseñanza, metodología del aprendizaje y maneras de evaluar.

Esta manera de entender la didáctica no es exclusiva de España, sino que en otros lugares se manifiesta un interés semejante. Así en el caso de Venezuela el profesor José A. Santiago reivindica "lo cotidiano como opción para obtener el conocimiento científico, pues ello facilita el estudio de los temas geográficos en sus escenarios naturales, y da crédito a la emisión de las concepciones de los actores protagonistas de los hechos geográficos vividos". E igual sucede en Colombia, donde en el campo de la educación geográfica se nota claramente "la necesidad de cambiar la forma tradicional de enseñar geografía, de tal manera que los niños y adolescentes la entiendan y apliquen en su vida cotidiana, despertando el sentido crítico y la formación ciudadana" ${ }^{48}$.

Estas coincidencias en la manera de ver la educación ciudadana desde la geografía fue lo que desembocó en la elaboración sobre un monográfico en relación con ambas materias. En dicho volumen reivindicamos el estudio de la ciudadanía como la pertenencia a un conjunto de valores que se entienden universales, como es la tolerancia, la responsabilidad de los actos y la búsqueda del bien

$46 \quad$ Nos referimos a los proyectos de investigación sobre la enseñanza de la Historia, que parte de los recuerdos del alumnado después de haber pasado más de un año de haber finalizado sus estudios de bachillerato. La citada investigación está dirigida por Nicolás Martínez, de la Universidad de Murcia, y entre sus resultados publicados podemos destacar la síntesis realizada en la revista Didáctica de las ciencias experimentales y sociales, (Martínez Valcárcel, Beltrán Llavador y Souto González, 2005 33-55).

47 Así en el caso del trabajo, (Ramírez y Souto, 2002) [http://www.ub.es/geocrit/sn/sn119137.htm] o de la vivienda [http://www.ub.es/geocrit/sn/sn-146(114).htm] (Dopazo, Menor y Souto, 2003).

48 Respecto al profesor José Santiago Rivera, hemos seguido sus trabajos publicados en Venezuela (Santiago, 2006), así como la exposición de sus ideas en Geocrítica (http://www.ub.es/geocrit/b3w-608.htm). Respecto a la referencia a la geografía colombiana he seguido el resumen realizado por Luis Aragón (2001) sobre el XVI Congreso Colombiano de Geografía (http://www.ub.es/geocrit/b3w-265.htm) 
público, que se plasman en diferentes lugares. Todo ello nos conduce a combinar diferentes escalas en la construcción de la ciudadanía, como es el hogar familiar o el territorio nacional.

Tal como señalaba en el primer capítulo de un reciente estudio monográfico sobre la formación ciudadana desde la geografía, entiendo que es posible desarrollar los valores fundamentales de la ciudadanía en su ámbito espacial atendiendo a los hechos cotidianos ${ }^{49}$. Para ello se hace preciso profundizar en los problemas sociales y en las metodologías didácticas. A este diagnóstico se ha venido a sumar recientemente el debate sobre las competencias básicas para la educación obligatoria.

Esto nos ha llevado a plantearnos qué puede aportar el concepto de competencias al actual desafío educativo de la formación básica. Entendemos, en primer lugar, que este tipo de estudio ya no puede quedar recluido en el ámbito de los escolares niños y adolescentes. Al menos desde las directrices que se derivan de la Estrategia de Lisboa y de las políticas gubernamentales, como la española, la formación permanente de los trabajadores está relacionada con la formación académica básica ${ }^{50}$. Ello incluso ha determinado que se adopten en toda la Unión Europea una jerga que llega a las aulas de todos los centros escolares, como son las competencias, que sustituyen al concepto de las cualificaciones.

En efecto, la Recomendación de la Unión Europea ${ }^{51}$ sobre las competencias básicas nos pone de relieve que este concepto incide en la formación permanente desde la perspectiva de la competitividad. Se trata de sustituir el concepto de las cualificaciones (resultados de aprendizaje) por éste, que refleja más exactamente el proceso de aprendizaje para alcanzar un determinado puesto laboral.

\section{NUEVOS RETOS E INVESTIGACIONES NECESARIAS}

La educación en el siglo XXI nos presenta un panorama bien diferente a lo que sucedía en la centuria anterior, en especial en los países europeos. Los nuevos recursos informáticos y de la comunicación inciden en la percepción que las personas tienen sobre el aprendizaje. Especialmente los adolescentes, que relacionan el acceso a estos recursos como la adquisición inmediata de un nuevo conocimiento. Igualmente las nuevas estructuras sociales familiares determinan la función de custodia escolar, lo que incide en la comunicación entre docentes y familias y también en el seno de la misma institución doméstica. Y, como hemos anotado, la incidencia del marco de la formación permanente, siguiendo los esquemas trazados en la Estrategia de Lisboa del año 2000.

No es posible realizar un inventario de todas las investigaciones que se derivan de los retos antes citados. Pero al menos queremos llamar la atención sobre tres ámbitos de investigación necesarios para afrontar las nuevas necesidades educativas.

49 La referencia corresponde al artículo: Espacio geográfico y educación para la ciudadanía (Souto, 2007; 11-31).

50 Por ejemplo ello se observa en la reglamentación de la formación para el empleo, aprobada en el año 2007, para integrar la antigua formación ocupacional y continua. Ver: REAL DECRETO 395/2007, de 23 de marzo, por el que se regula el subsistema de formación profesional para el empleo.

51 Recomendación del Parlamento Europeo y del Consejo, de 18 de diciembre de 2006, sobre las competencias clave para el aprendizaje permanente (2006/962/CE) 
En primer lugar será preciso abordar desde investigaciones empíricas las características de la diversidad de situaciones personales en el aprendizaje permanente. Será preciso conocer las técnicas de trabajo docente que facilitan el aprendizaje de los alumnos. Un ámbito que se relaciona con la necesidad de transformar los actuales planes de formación inicial del profesorado: en este sentido harían falta más trabajos donde se relacione la enseñanza y el aprendizaje, tal como mostró en su investigación $\mathrm{K}$. Bain y que ha sido objeto de una importante reseña en Biblio3 $W^{52}$.

En segundo lugar será preciso explicar las funciones escolares en la sociedad actual. Los estudios genéticos sobre el sistema escolar han incidido en los factores históricos que condicionaron la organización del curriculum, así como las instituciones de control del saber escolar. En este siglo XXI será necesario abordar las relaciones que se establecen entre la institución escolar y los entornos sociales en que éste se sitúa. El papel de los movimientos sociales que han conformado lo que se denomina comunidad escolar, o más específicamente comunidades de aprendizaje será un objeto de estudio necesario para afrontar los cambios educativos y sociales. E igualmente la definición de las características de cómo se comunican y aprenden los adolescentes, un concepto cultural que encierra mucha hipocresía social.

Por último deberemos abordar el papel de las materias escolares y su incidencia en la creación de una cultura escolar pública. Las didácticas específicas han estudiado el papel del conocimiento científico en el saber escolar y han desarrollado conceptos tales como la trasposición didáctica. Frente a estas tendencias analíticas se ha propuesto el estudio integrado de los problemas educativos. Es necesario recuperar el papel del conocimiento científico en el saber científico si no queremos correr el riesgo de transformar el conocimiento escolar en un saber superficial.

Lo que nos debe preocupar a los profesionales de la educación geográfica es cómo traducir las competencias básicas en saberes que se puedan movilizar de forma integrada, o sea no yuxtapuesta, en la resolución de situaciones de la vida cotidiana: diseñar un viaje, buscar un barrio para vivir, un trabajo, la participación en la vida democrática, analizar una propuesta de planeamiento urbano, etc. Es decir, no se trata de hacer un listado de conceptos y hechos, ni tampoco una selección de técnicas de trabajo, sino de buscar el desarrollo de competencias geográficas que sean útiles a la formación ciudadana.

A este respecto también encontramos en la revista Geocrítica una buena referencia a los cambios acaecidos en el conocimiento geográfico y su incidencia en la formación ciudadana. Nos referimos a la sección de Ciudadanía y participación que dirige Liliana Fracasso y que cuenta con las secciones de: participación en la elaboración de planes generales, participación en planes parciales, presupuesto participativo y seguimiento y control de los planes $^{53}$.

Para conocer las preocupaciones de los geógrafos respecto a la enseñanza de la materia hemos analizado los contenidos de los diferentes congresos organizados por el Grupo de Didáctica de la Asociación de Geógrafos Españoles (AGE). Pretendíamos así comparar las preocupaciones de los ponentes y comunicantes con las posibles demandas del profesorado en el aula (ver cuadro 1).

52 Nos referimos a la reseña de los profesores A. Luis y J. Romero (2006) [http://www.ub.es/geocrit/b3w-652.htm] en relación con el trabajo de K. Bain (2005) 
Cuadro 1. Congresos del Grupo de Didáctica de la AGE.

\begin{tabular}{|c|c|c|c|}
\hline $\begin{array}{c}\text { Actividades del } \\
\text { Grupo de Didáctica } \\
\text { de la AGE Jornadas }\end{array}$ & Tema central & Ponencias & $\mathrm{N}^{\mathrm{o}}$ comunicaciones \\
\hline I. Madrid, 1988 & $\begin{array}{l}\text { Demandas de la } \\
\text { geografía ante la } \\
\text { Reforma LOGSE }\end{array}$ & $\begin{array}{l}\text { Reformas planes estudio. } \\
\text { Didáctica activa. Geografía y } \\
\text { ciencias sociales }\end{array}$ & $\begin{array}{l}45 \text { ( } 22 \text { en la sección } \\
\text { didáctica activa) }\end{array}$ \\
\hline II. Burgos, 1991 & Geografía y Reforma & $\begin{array}{l}\text { Geografía en el diseño } \\
\text { curricular. Geografía y } \\
\text { Educación ambiental }\end{array}$ & $\begin{array}{l}19 \text { (13 en la sección } \\
\text { de educación } \\
\text { ambiental) }\end{array}$ \\
\hline III. Madrid, 1996 & $\begin{array}{l}\text { Geografía y el reto } \\
\text { educativo en el marco } \\
\text { de la Reforma }\end{array}$ & $\begin{array}{l}\text { Los procedimientos. El paisaje } \\
\text { como referencia. Temas } \\
\text { transversales y geografía }\end{array}$ & $\begin{array}{l}46 \text { ( } 19 \text { en la sección } \\
\text { del paisaje) }\end{array}$ \\
\hline IV. Alicante, 1998 & Educación y Geografía & $\begin{array}{l}\text { El espacio como objeto de } \\
\text { enseñanza. Los valores en } \\
\text { le medio urbano. Recursos } \\
\text { didácticos y medio rural }\end{array}$ & $\begin{array}{l}54 \text { ( } 23 \text { en la sección } \\
\text { de valores) }\end{array}$ \\
\hline V. Murcia, 2000 & $\begin{array}{l}\text { Geografía, profesorado } \\
\text { y sociedad }\end{array}$ & $\begin{array}{l}\text { Formación del profesorado. } \\
\text { Innovación didáctica. } \\
\text { Contenidos científicos }\end{array}$ & $\begin{array}{l}43 \text { ( } 21 \text { en la sección } \\
\text { de innovación } \\
\text { didáctica) }\end{array}$ \\
\hline VI. Toledo, 2003 & $\begin{array}{l}\text { Las nuevas demandas } \\
\text { sociales }\end{array}$ & $\begin{array}{l}\text { Inmigración e } \\
\text { interculturalismo. Las nuevas } \\
\text { tecnologías. Las propuestas de } \\
\text { la LOCE }\end{array}$ & $\begin{array}{l}42 \text { (16 en la sección } \\
\text { de innovación } \\
\text { didáctica) }\end{array}$ \\
\hline VII. Valencia, 2007 & $\begin{array}{l}\text { Las competencias } \\
\text { geográficas y } \\
\text { ciudadanía }\end{array}$ & $\begin{array}{l}\text { Sostenibilidad; urbanismo } \\
\text { local y global; migraciones e } \\
\text { interculturalidad }\end{array}$ & $\begin{array}{l}31 \text { (13 en la sección } \\
\text { de sostenibilidad) }\end{array}$ \\
\hline VIII. Jaén, 2008 & $\begin{array}{l}\text { La cultura geográfica } \\
\text { en la era de la } \\
\text { globalización }\end{array}$ & $\begin{array}{l}\text { Formación docente, } \\
\text { innovaciones didácticas e } \\
\text { investigación aplicada }\end{array}$ & s.d. \\
\hline IX. Madrid 2010 & $\begin{array}{l}\text { Geografía, educación } \\
\text { y formación del } \\
\text { profesorado en el marco } \\
\text { del Espacio europeo de } \\
\text { Educación Superior }\end{array}$ & $\begin{array}{l}\text { Formación del profesorado. } \\
\text { Innovación didáctica e } \\
\text { interculturalidad }\end{array}$ & $\begin{array}{l}57 \text { comunicaciones } \\
\text { de diversas } \\
\text { aportaciones sobre } \\
\text { formas de aprender } \\
\text { geografía }\end{array}$ \\
\hline
\end{tabular}

Tal como se desprende del cuadro anterior, a los profesionales de la didáctica de la geografía les preocupa en especial aquellas comunicaciones que pueden incidir en la innovación didáctica en el aula. Sin embargo, el eco de sus propuestas ha sido muy escaso. Por eso es necesario cuestionarse los motivos de dicha separación entre la teoría universitaria y la práctica de la enseñanza básica, pues las experiencias no llegan al aula. 
En consecuencia, las innovaciones han sido gestadas en el trabajo voluntarista, en algún caso con apoyo institucional de los programas de reforma de enseñanza y de los centros de profesores, por equipos de profesores de secundaria: Asklepios, Aula Sete, Cronos, Gea-Clío, Ínsula Barataria, Ires, Kairós y Pagadí. Grupos que modifican sus biografías personales por incorporación de algunos de sus miembros a las universidades y que se acaban integrando en la plataforma Fedicaria, que después de algunos años de vida acaba disgregándose en diferentes proyectos particulares: Nebraska, grupos locales y la continuidad de Gea-Clío.

No es posible, ni tampoco es su objetivo, analizar la deriva de los diferentes proyectos curriculares en España, sino más bien estudiar algunos resultados para avanzar en la posible confluencia entre investigación universitaria y acción docente en la enseñanza básica. Y como hemos hecho en otras ocasiones vamos a recurrir a la experiencia personal dentro del proyecto Gea-Clío.

\section{LAS POSIBILIDADES DE UN PROYECTO CURRICULAR}

Como hemos subrayado en páginas precedentes creemos que la alternativa a la cesura entre las investigaciones geográficas educativas y las innovaciones en la geografía escolar reside en la elaboración de buenos proyectos curriculares. Sin embargo ello es difícil, pues elaborar un proyecto curricular supone cohesionar un grupo de intereses personales y disponer de recursos para difundir las ideas. Y al menos esta labor supone tres años de trabajo constante. Y no siempre comprendido por la comunidad académica y escolar.

Tal como señalaba hace unos años el profesor José Gimeno (1989) el "concepto de proyecto curricular es de uso muy reciente y poco extendido entre nosotros (los españoles), por lo que es conveniente hacer alguna precisión sobre el mismo.... se alude a una forma o método didáctico de organizar el trabajo de los alumnos en la escolarización o, si se quiere, a una manera de vertebrar el currículum en términos de tareas prácticas.... Los proyectos curriculares son elaboraciones cuidadas de los contenidos de una parcela o área del currículum que se plasman en materiales de cierta calidad para profesores y alumnos" (Gimeno, 1989: 14-18).

Es decir, para editar materiales es preciso disponer de una sólida reflexión teórica sobre la metodología didáctica, los conocimientos técnicos de la materia y las posibilidades de desarrollo curricular. Por eso entendimos que la publicación de materiales implicaba un esfuerzo en la formación del profesorado. Para ello centramos los esfuerzos en la construcción de libros destinados al docente, para que dispusiera de unas orientaciones para mejorar su práctica profesional.

Tal como recordaba el antedicho profesor Gimeno, un proyecto de este tipo es "algo que no está al alcance de todos los profesores y menos de los equipos que componen el profesorado de cualquier centro" (Gimeno, 1989: 14-18). Era preciso disponer de una ayuda institucional y editorial; en nuestro caso fue el programa de reforma y formación del profesorado de la Generalitat Valenciana entre 1989 y 1996 y el apoyo de la editorial Nau Llibres. Este enorme esfuerzo pretendía colocar al alcance de los profesores un material y unas ideas para elaborar el proyecto educativo de su centro, pero sin necesidad de hacer su proyecto curricular propio. Creemos y así lo muestra la experiencia acumulada, que es un enorme error asignar unas tareas desproporcionadas a los profesores en sus centros escolares, sobre todo si no se transforma el reparto de tareas horarias. Para innovar es preciso disponer de un tiempo para reflexionar y trabajar conjuntamente y no sólo con los compañeros del departamento y centro, sino con otros foráneos que pueden aportar nuevas ideas. 
En el cuadro 2 hemos seleccionado algunas de las temáticas que hemos desarrollado en los libros del profesorado editados por el proyecto Ga-Clío para Secundaria Obligatoria. El objetivo era ofrecer al profesorado unas lecturas que le permitieran construir su propio curriculum escolar. Las sesiones de trabajo, a modo de seminarios didácticos, nos han permitido valorar la traslación de estas finalidades a las tareas escolares. Aunque es cierto que era posible incidir más en la práctica, lo cierto es que los veinte años que han transcurrido desde el inicio del proyecto hasta la actualidad manifiestan que algunas innovaciones sí se han producido en el campo de la enseñanza de la geografía en la Secundaria Obligatoria y Bachillerato en Valencia y España.

Cuadro 2. Ejemplo de algunos libros del profesor de Gea-Clío en Secundaria Obligatoria.

\begin{tabular}{|c|c|c|}
\hline Título del libro & Orientación didáctica & Actualización científica \\
\hline $\begin{array}{l}\text { Espacio subjetivo y geografía. } \\
\text { Orientación teórica y praxis didáctica }\end{array}$ & $\begin{array}{l}\text { Geografía y espacio subjetivo } \\
\text { en España. Compilación de los } \\
\text { principales trabajos y análisis } \\
\text { crítico }\end{array}$ & $\begin{array}{l}\text { Espacio subjetivo y geografía. } \\
\text { Consideraciones teóricas y } \\
\text { metodológicas }\end{array}$ \\
\hline $\begin{array}{l}\text { El estudio geográfico de la población. } \\
\text { Orientación teórica y praxis didáctica. }\end{array}$ & La evaluación en geografía & $\begin{array}{l}\text { Geografía de la población. } \\
\text { Tendencias recientes. }\end{array}$ \\
\hline $\begin{array}{l}\text { Historia y escuela. La escuela como } \\
\text { objeto de estudio de la historia } \\
\text { enseñada. Orientación teórica y } \\
\text { praxis didáctica. }\end{array}$ & $\begin{array}{l}\text { La fuente oral: un recurso a } \\
\text { nuestro alcance }\end{array}$ & $\begin{array}{l}\text { La constitución de los } \\
\text { sistemas escolares públicos. } \\
\text { Notas históricas }\end{array}$ \\
\hline $\begin{array}{l}\text { Arqueología, hominización, } \\
\text { sociedades cazadoras-recolectoras. } \\
\text { Orientación teórica y praxis didáctica. }\end{array}$ & $\begin{array}{l}\text { La utilización de materiales } \\
\text { audiovisuales y literarios en la } \\
\text { unidad didáctica }\end{array}$ & $\begin{array}{l}\text { Arqueología: proceso de } \\
\text { hominización, sociedades } \\
\text { cazadoras-recolectoras }\end{array}$ \\
\hline $\begin{array}{l}\text { Discriminaciones sociales y étnicas en } \\
\text { un mundo intercultural y complejo. } \\
\text { Orientación teórica y praxis didáctica. }\end{array}$ & $\begin{array}{l}\text { Valores democráticos en un } \\
\text { mundo intercultural }\end{array}$ & $\begin{array}{l}\text { Actitudes y valores para la } \\
\text { formación en el respeto y la } \\
\text { tolerancia }\end{array}$ \\
\hline $\begin{array}{l}\text { El mundo árabe y el Mediterráneo. } \\
\text { Orientación teórica y praxis didáctica. }\end{array}$ & $\begin{array}{l}\text { El Islam, el mundo árabe, } \\
\text { el Magreb en los manuales } \\
\text { españoles. Entre lo que es y lo } \\
\text { que debería ser. }\end{array}$ & $\begin{array}{l}\text { Crisis global y emergencia de } \\
\text { los movimientos islamistas en } \\
\text { el Magreb }\end{array}$ \\
\hline $\begin{array}{l}\text { La Historia como interpretación } \\
\text { de conflictos. Orientación teórica y } \\
\text { praxis didáctica. }\end{array}$ & Bibliografía comentada & $\begin{array}{l}\text { Demografía, sociedad rural } \\
\text { y agricultura en la Europa } \\
\text { moderna }\end{array}$ \\
\hline
\end{tabular}

Como podemos comprobar los retos de la educación geográfica en este siglo XXI que estamos iniciando son complejos y requieren de una profunda investigación sobre los objetivos de la didáctica escolar y el funcionamiento del sistema de aprendizaje permanente. Algo que está ausente de la mayoría de las preocupaciones de los gremios de geógrafos y universidades. Y con improvisaciones es difícil dar una respuesta útil a las demandas sociales de una educación que sea útil para las expectativas personales. De tal modo que la pregunta inicial ¿por qué estudiar geografía?, debería transformarse en: ¿por qué es necesario investigar en la educación geográfica? 


\section{BIBLIOGRAFÍA}

Aragón, L. E. (2001). Por la construcción de un proyecto territorial nacional [En línea] Biblio $3 W$. Revista Bibliográfica de Geografia y Ciencias Sociales (265).

Araya Palacios, F. (2009). Enseñanza, investigación y conocimiento geográfico en contextos escolares formales y no formales [En línea]. Biblio 3W. Revista Bibliográfica de Geografia y Ciencias Sociales (831).

Bain, K. (2005). Lo que hacen los mejores profesores de universidad, Valencia: Publicacions de la Universitat de València.

Ballester Vallori, A. (1999). La didàctica de la geografia. Aprenentatge significatiu i recursos didàctics de les Illes Balears. Mallorca: Edicions Documenta Balear.

Barreiro Paradela, L. (1936). Nova Xeografia de Galicia pras escolas, Santiago: Editorial P.A.G. (edición facsímil de 1980 por Editorial Sálvora).

Calandra, L. M. (2008). Il territorio attraverso le carte geografiche: un modello didattico per la scuola di base. [En línea]. Scripta Nova, Revista Electrónica de Geografia y Ciencias Sociales, 270 (124).

Capel, H. (1998, 15 de abril). Una geografía para el siglo XXI. [En línea] Scripta Nova, Revista Electrónica de Geografia y Ciencias Sociales (19).

Claudino, S. L. N. (2001). Portugal através dos manuais escolares de Geografia. Século XIX. As imagens intencionais. (Tese de doutoramento). Faculadade de Letras, Lisboa.

Claudino, S. Rodrigo Oliveira, A. (2005, 10 de junio). A cidadania na formação de professores de Geografia em Portugal e Brasil. [En línea] Biblio 3W, Revista Bibliográfica de Geografía y Ciencias Sociales (588).

Dopazo, I.; Menor, S.; Souto, X. M. (2003). La vivienda como problema educativo [En línea] Scripta Nova, Revista Electrónica de Geografia y Ciencias Sociales, 146(114).

García Pérez, F. (1999). El medio urbano en la educación secundaria obligatoria. Las ideas de los alumnos y sus implicaciones curriculares. (Tesis Doctoral). Universidad de Sevilla, Facultad de Ciencias de la Educación, Departamento de Didáctica de las Ciencias Sociales y Experimentales, Sevilla.

García Pérez, F. (2003). Las ideas de los alumnos y la enseñanza del medio urbano. Sevilla: Díada Editora, S. L.

García Pérez, F.; Porlán Ariza, R. (2000). El proyecto IRES (Investigación y Renovación Escolar) [En línea] Biblio 3W. Revista Bibliográfica de Geografia y Ciencias Sociales (205).

Gimeno Sacristán, J. (1989). Proyectos curriculares. ¿Posibilidad al alcance de los profesores?, Cuadernos de Pedagogía, (172), 14-18. 
Guijarro Fernández, A. (1997). El diseño curricular de Ciencias Sociales en la educación secundaria obligatoria. Fundamentos de las propuestas de articularlo en torno al estudio de problemas actuales. (Tesis Doctoral). Universidad de Cantabria, Departamento de Ciencias de la Educación, Santander.

Luis, A. y Romero, J. (2006). Sabedores de lo aceptado, estudiantes bulímicos y pensadores independientes: De la Universidad de la enseñanza a la del aprendizaje. [En línea] Biblio 3W. Revista Bibliográfica de Geografia y Ciencias Sociales (652).

Luis, A. y Romero, J. (2007). Escuela para todos, conocimiento académico y geografía escolar en España (1830-1953). Santander: Servicio de Publicaciones de la Universidad de Cantabria.

Martínez Valcárcel, N.; Souto González, X. M. y Beltrán Llavador, J. (2005). Los problemas docentes en historia de Bachillerato: la gran diversidad bajo la aparente uniformidad. Didáctica de las ciencias experimentales y sociales, (19), 33-55.

Melcón Beltrán, J. (1989). La geografía y la formación de maestros en España, 1836-1914. Geocrítica, (83).

Melcón Beltrán, J. (1995). La renovación de la enseñanza de la geografia en los orígenes de la España contemporánea. Cuadernos del ICE, número 14. Madrid: Ediciones de la Universidad Autónoma de Madrid.

Miquel Albert, J. y Alegre, E. (2004). Internet i Ciències Socials. Una experiència de comunitat virtual a l'ensenyament secundari. [En línea]. Geocrítica / Scripta Nova, Revista Electrónica de Geografia y Ciencias Sociales, 170 (67).

Ramírez, S. y Souto, X. M. (2002). El trabajo y la didáctica de la geografía e historia [En línea] Scripta Nova, Revista Electrónica de Geografia y Ciencias Sociales, 119 (137).

Ramiro Roca, E. (1998). La institucionalització i difusió de la geografia escolar: la percepció del profesorat de la Comarca de la Ribera. (Tesis Doctoral). Universidad de Valencia, Facultad de Geografía e Historia, Valencia.

Ramiro Roca, E. (1998). ¿Evolucionamos o nos quedamos quietos? Los recursos utilizados en la clase de geografía. [En línea]. Scripta Nova, Revista Electrónica de Geografia y Ciencias Sociales (29).

Rodríguez de Moreno, E. A. (2000). Geografia Conceptual. Enseñanza y aprendizaje de la geografia en la Educación Básica Primaria. Bogotá: Tercer Mundo Editores.

Romero Morante, J. (1998). Recursos informáticos al servicio de una socialización crítica. Una propuesta de utilización en la enseñanza aprendizaje de la Historia para la educación secundaria. (Tesis Doctoral). Universidad de Oviedo, Facultad de Pedagogía.

Santiago Rivera, J. A. (2005). La geografía descriptiva en los programas escolares de la enseñanza geográfica en Venezuela. [En línea]. Biblio 3W, Revista Bibliográfica de Geografia y Ciencias Sociales, $\mathrm{X}(608)$. 
Santiago Rivera, J. A. (2006). Otras formas para enseñar y aprender geografía en la práctica escolar. Revista de Pedagogía, 27(80).

Santiago Rivera, J. A. (2008, 15 de febrero). Evolución histórica de la enseñanza de la geografía en Venezuela. [En línea]. Geografia Digital, 3(5).

Santos Da Silva Alberto, A. F. (2000). O Contributo da educação geográfica na educação ambiental. A Geografia no Ensino Secundàrio. (Tese de Mestrado em Geografia Humana). Faculdade de Letras, Lisboa.

Santos Da Silva Alberto, A. F. (2002). O contributo da educação geográfica na Educação Ambiental. O caso da Geografia no Ensino Secundário. [En línea]. Scripta Nova, Revista Electrónica de Geografia y Ciencias Sociales (114).

Solbes, J. Souto, X. M. y Traver, M. J. (2004). El impacto de las nuevas tecnologías de la información y de la comunicación en el sistema escolar. [En línea]. Scripta Nova, Revista Electrónica de Geografia y Ciencias Sociales, 170(71).

Souto González, X. M. (1988). Geografia y ciencias sociales. Propuesta de diseño curricular 12-16 años, Valencia: Generalitat Valenciana. Direcció General d'ordenació i innovació educativa, Gráficas Vimar.

Souto González, X. M. (2001). Cultura académica, fracaso escolar y reforma de las humanidades [En línea]. Scripta Nova, Revista Electrónica de Geografia y Ciencias Sociales (96).

Souto González, X. M. (2001). Educación, identidad y geografía: una propuesta para profesores y alumnos desde Colombia. [En línea]. Biblio 3W Revista Bibliográfica de Geografía y Ciencias Sociales (292).

Souto González, X. M. (2002). Coñecemento xeográfico e cultura escolar. [En línea] Biblio 3W, Revista Bibliográfica de Geografia y Ciencias Sociales (379).

Souto González, X. M. (2006). Geografía en Argentina. [En línea] Biblio 3W Revista Bibliográfica de Geografia y Ciencias Sociales (640).

Souto González, X. M. (2007). Espacio geográfico y educación para la ciudadanía. Revista Didáctica Geográfica (9).

Villanueva Zarazaga, J. (2003) Geografia y Educación Secundaria. De la guerra civil a la democracia, Zaragoza: Gobierno de Aragón, Colección Aragón en el Aula.

Artículo recibido 21 - 09 - 10. Aprobado 26 - $11-10$. 